Article

\title{
Hybrid Composites Based on Polypropylene with Basalt/Hazelnut Shell Fillers: The Influence of Temperature, Thermal Aging, and Water Absorption on Mechanical Properties
}

\author{
Anna Kufel *(D) and Stanisław Kuciel (D) \\ Faculty of Materials Engineering and Physics, Institute of Materials Engineering, Tadeusz Kosciuszko Cracow \\ University of Technology, Al. Jana Pawła II 37, 31-864 Cracow, Poland; stask@mech.pk.edu.pl \\ * Correspondence: anna.kufel93@gmail.com; Tel.: +48-667-046-051
}

Received: 15 November 2019; Accepted: 17 December 2019; Published: 20 December 2019

\begin{abstract}
The aim of the research was to study the effects of adding natural fillers to a polypropylene (PP) matrix on mechanical and physical properties of hybrid composites. The $10 \%, 15 \%$, and $20 \%$ by weight basalt fibers (BF) and ground hazelnut shells (HS) were added to the PP matrix. Composites were produced by making use of an injection molding method. Tensile strength, tensile modulus, strain at break, Charpy impact strength, and the coefficient of thermal expansion were determined. The influence of temperature, thermal aging, and water absorption on mechanical properties was also investigated. In addition, short-time creep tests were carried out. To characterize the morphology and the filler distribution within the matrix, a scanning electron microscope (SEM) was used. The results showed that the addition of the two types of filler enhanced mechanical properties. Furthermore, improvements in thermal stability were monitored. After water absorption, the changes in the tensile properties of the tested composites were moderate. However, thermal aging caused a decrease in tensile strength and tensile modulus.
\end{abstract}

Keywords: hazelnut shell; basalt fiber; polypropylene; hybrid composites; natural filler; thermoplastic

\section{Introduction}

In recent years, natural fibers have increasingly been used as an alternative to traditional synthetic fibers, which have harmful effects on the environment. Furthermore, agricultural waste is used to manufacture composites due to an increasing demand for eco-friendly materials. Composites may be reinforced (e.g., by wheat husk and rye husk [1], rice husk [2], cocoa pod husk [3], or spent coffee ground [4]). Walnut shells are renewable lignocellulosic materials and they can be obtained as agricultural by-products. In 2017, the world production of hazelnuts (in shells) was 1 million tonnes [5]. Hazelnut shells (HSs) are characterized by low density; as reported by Copur [6], the density of hazelnut shells is $0.23 \mathrm{~g} / \mathrm{cm}^{3}( \pm 0.21)$, which appears to be a lower value compared to raw wood material $\left(0.40-0.75 \mathrm{~g} / \mathrm{cm}^{3}\right)$. Gurbanow et al. [7] studied polypropylene (PP) composites with hazelnut shell flour. It was found that HSs can be used as a filler in polymer composites. In another study [8], HSs were used in bio-based composites based on polylactic acid. The addition of HSs increased the stiffness of the composites. Among various natural fibers, basalt fibers (BFs) have been extensively used in various fields of engineering. BFs are mineral fibers with mechanical properties, similar to glass fibers, and low-cost compared to carbon fibers [9]. BFs have good physical, chemical, and thermal properties as well as excellent mechanical and chemical resistance. Basalt is a natural material, and no chemical additives, solvents, pigments, or other hazardous materials, are used in the production of its fibers. Caused by that fact, BF can be considered a sustainable material [10]. Composites 
reinforced with BFs are widely used due to their high mechanical properties and thermal stability; additionally, they are eco-friendly materials. Many researchers have studied composites reinforced by BFs [11-13]. Manikandan et al. [14] investigated the effect of surface modifications of BFs on mechanical properties. The results showed that acid treatment of BFs improved tensile strength and impact strength. Yang et al. [15] added carbon nanotubes and aluminum hypophosphite to polylactide/BF composites, which reduced the peak heat release rate of the composite. Hybrid composites offer better balance in mechanical properties than non-hybrid composites. Predicting their mechanical properties is challenging due to the synergistic effects between both fibers [16]. Hybridization provides us with the opportunity to improve some of the properties of composites. It is also common to reduce production costs by replacing the more expensive filler with a cheaper filler. By using hybridizations, composites can be produced that combine the features of both fillers. An addition of carbon fibers with smaller diameters and larger and longer BFs to polyamide 6, improved mechanical properties of composites [17]. Mechanical properties of natural filler composites are lower compared to synthetic fibers. Furthermore, natural fillers absorb moisture. The amalgamation of two different types of fillers in the same matrix can result in a material that overcomes these drawbacks. A few studies reported hybrid composites reinforced by both natural and synthetic fillers. Samal et al. [18] studied hybrid PP composites with banana and glass fibers. In another study [19], mechanical properties and the water absorption of high-density polyethylene composites, reinforced by wood and carbon fibers, were investigated. An addition of a small amount of carbon fibers to wood plastic composites enhanced the mechanical properties without additional operating costs. In another study [20], effects on mechanical performance of composites with man-made cellulose and glass fibers were investigated.

There is research on composites reinforced with HSs and BFs; however, hybrid composites with these fillers have not been studied so far. This study aimed to investigate the influence of an addition of natural fillers to a polypropylene matrix on mechanical properties. Furthermore, the effect of temperature, water absorption, and thermal aging was studied. Additionally, such properties as thermal conductivity, thermal expansion, and creep behavior were monitored. The characterization of an adhesion of fillers with the matrix by scanning electron microscope (SEM) was observed.

\section{Materials and Methods}

\subsection{Materials}

PP Moplen HP500N produced by Basell Orlen Polyolefins (Płock, Poland) was used as a matrix. The following fillers and coupling agents were used:

1. The chopped basalt fibers (BCS17-6.4-KV16) with a cutting length of $6.4 \mathrm{~mm}$ and a nominal diameter of $17 \mu \mathrm{m}$ were supplied by Basaltex (Wevelgem, Belgium).

2. Hazelnut shells were ground on a Retsch EMAX mill (Retsch GmbH, Haan, Germany). The particle size was from 90 to $200 \mu \mathrm{m}$. The shells were treated with $5 \% \mathrm{NaOH}$ for $1 \mathrm{~h}$, rinsed with water, and dried for $2 \mathrm{~h}$ at $90^{\circ} \mathrm{C}$ in a molecular drier.

3. The maleic anhydride PP (MAPP) PP SCONA TPPP 9112 GA was supplied by Byk (Altana AG, Wesel, Germany).

\subsection{Methods of Testing}

\subsubsection{Preparation of Composite Specimens}

Composites were prepared in the Laboratory of Plastics Technology operating under Grupa Azoty S.A. in Tarnów, Poland. The BFs, HSs, and PP were compounded on a MARIS TM30 co-rotating twin-screw extruder (Maris America Corp., Windsor Mill, MD, USA). The samples were made by using an Engel ES 200/40 HSL injection-molding machine (ENGEL GmbH, Schwertberg, Austria). The following parameters were set: temperature from 180 to $220^{\circ} \mathrm{C}$, injection speed from 60 to $90 \mathrm{~mm} / \mathrm{s}$, rotation speed of the screw of $63 \mathrm{rpm}$, a cycle time of $60 \mathrm{~s}$, and a mold temperature of $40^{\circ} \mathrm{C}$. 


\subsubsection{Density}

The density (PN-EN ISO 1183) of the samples was estimated using the hydrostatic method on a RADWAG WAS 22W scale (Radwag, Radom, Poland). The specimens were measured in an ethanol medium.

\subsubsection{Vicat Softening Temperature Measurement}

The softening temperature was tested with a Ceast Heat Deflection Temperature (HDT) and Vicat Tester Type 6520 (CEAST, Pianezza, Italy) machine. The measurements were done using method A50, according to PN-EN ISO 306:2006. The $10 \mathrm{~N}$ was applied. The bath temperature was raised to $50^{\circ} \mathrm{C} / \mathrm{h}$ until the needle penetrated $1 \mathrm{~mm}$.

\subsubsection{Mechanical Testing}

The mechanical properties were analyzed using an MTS Criterion Model 43 universal testing machine (MTS Systems Corp., Eden Prairie, MN, USA) with an MTS 634.31F axial extensometer, according to PN-EN ISO 527-1:2012. The constant cross-head speed was set to $5 \mathrm{~mm} / \mathrm{min}$. Charpy impact tests (PN-EN ISO 179-2) were carried out on unnotched samples using the Zwick/Roell MTS SP (Zwick Roell Group, Ulm, Germany) testing machine. Furthermore, the tests were performed after 30 days of water absorption, $144 \mathrm{~h}$ of aging, and at various temperatures $\left(-24,23\right.$, and $\left.80^{\circ} \mathrm{C}\right)$.

\subsubsection{Thermal Conductivity}

The thermal conductivity was measured with the hot disk thermal constants analyzer transient plane source (TPS) 500 (Hot Disk AB, Göteborg, Sweden) in accordance with ISO 22007-2.

\subsubsection{Thermal Expansion}

The analysis of composites was carried out on the NETZSCH 402 F1 Hyperion device (NERZSCH Group, Selb, Germany). The samples were placed vertically. The test parameters were recorded with Proteus software. The test temperature was between -60 and $140{ }^{\circ} \mathrm{C}$. The heating and cooling was set at $10^{\circ} \mathrm{C} / \mathrm{min}$. The coefficient of linear thermal expansion (LTCE) was computed using the following formula:

$$
\propto_{L}=\frac{1}{L} \frac{d L}{d T}, 1 / K
$$

where $L$ is the linear dimension of the specimen and $\mathrm{d} L / \mathrm{d} T$ is the rate at which the linear dimension changes per unit of temperature.

\subsubsection{Creep Test}

The short-time creep tests were performed using an electromechanical Zwick 2.5 testing machine (Zwick Roell Group, Ulm, Germany), according to PN-EN ISO 899-1:2005. Creep experiments at $23^{\circ} \mathrm{C}$ were carried out under an applied stress of $15 \mathrm{MPa}$.

\subsubsection{Composite Morphology}

The microstructure observations were made with the use of a scanning electron microscope JEOL JSN5510LV (JEOL Ltd., Tokyo, Japan). SEM structure images were acquired on tensile-test fracture surfaces of specimens. The accelerating voltage was $20 \mathrm{kV}$. Samples were coated with gold using a Cressington 108 auto sputter coater (Cressington Scientific Instruments, Watford, UK). 


\subsubsection{Water Absorption}

Water absorption, after soaking in distilled water for 1, 7, 20, and 30 days, was carried out according to the ASTM D570-98 standard. An electronic weighing balance (RADWAG WAS 22W) was used. The following equation was used to calculate the water absorption $(\% W)$ :

$$
\% W=\frac{W_{n}-W_{0}}{W_{0}} \times 100
$$

where $W_{0}$ and $W_{\mathrm{n}}$ is the weight of the sample before and after soaking in water, respectively; \%W is the percentage increase in weight.

\subsubsection{Thermal Aging}

Accelerated aging tests were realized using an autoclave (Parr Instrument Company, Moline, IL, USA) according to the EN ISO 2440 standard. Samples were conditioned for $16 \mathrm{~h}$ in the temperature of $23 \pm 2{ }^{\circ} \mathrm{C}$ and a humidity that was equal to $50 \pm 5 \%$. Temperature was set at $120{ }^{\circ} \mathrm{C}$, the humidity was $100 \%$, and the pressure was $0.3 \mathrm{MPa}$. The aging process lasted for $144 \mathrm{~h}$. A diagram of the accelerated aging process is shown in Figure 1. The arrangement of the samples in the autoclave is shown in Figure 2.

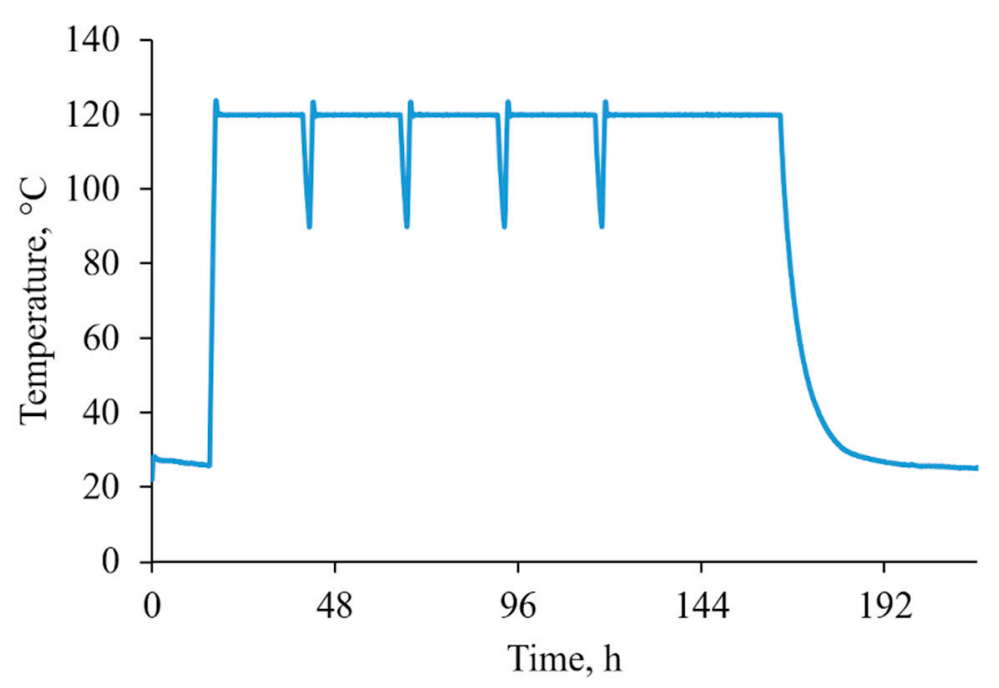

Figure 1. Diagram of the accelerated aging process in an autoclave.

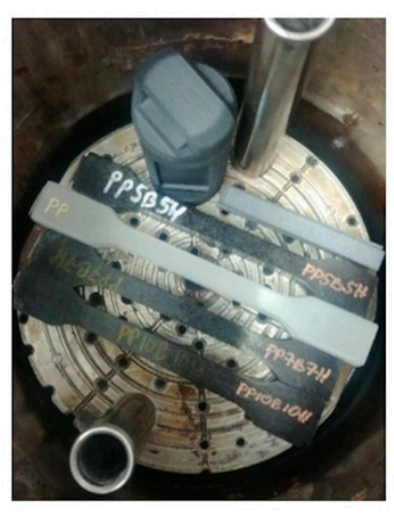

(a)

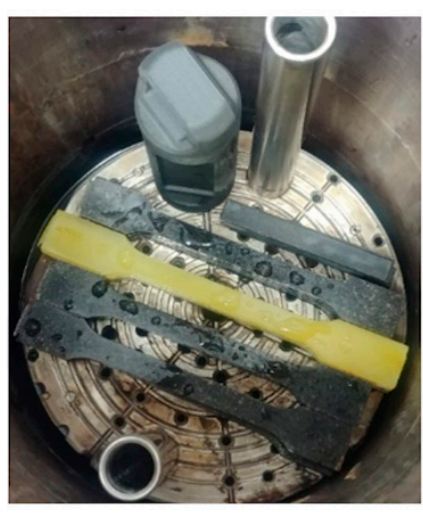

(b)

Figure 2. The appearance and arrangement of samples in an autoclave (a) before and (b) after the accelerated aging process. 


\section{Results and Discussion}

\subsection{Physical and Processing Properties}

Three different ratios were used to produce composites. A detailed description of the composition with the density measurement and Vicat softening temperature is given in Table 1 . The addition of fibers caused a slight increase in the density of composites. The density of composites containing $20 \mathrm{wt} \%$ fillers was $12.5 \%$ higher than for unfilled PP. However, hybrid composites with BFs and HS particles are still lightweight materials. The softening point was also determined. The Vicat softening temperature increased up to $161.8^{\circ} \mathrm{C}$ for the PP10B10H composite.

Table 1. Composition and basic properties of tested composites.

\begin{tabular}{|c|c|c|c|}
\hline Symbol & Composition & Density, $\mathrm{g} / \mathrm{cm}^{3}$ & Vicat Softening Temperature, ${ }^{\circ} \mathrm{C}$ \\
\hline PP & $100 \%$ polypropylene $\mathrm{HP} 500 \mathrm{~N}$ & 0.886 & 150.6 \\
\hline PP5B5H & $87 w t \%$ PP + $5 w t \%$ BF + $5 w t \%$ HS + $3 w t \%$ MAPP & 0.992 & 158.0 \\
\hline PP7B7H & $82 w t \% P P+7.5 w t \%$ BF $+7.5 w t \%$ HS $+3 w t \%$ MAPP & 0.993 & 161.2 \\
\hline PP10B10H & $77 w t \% P P+10 w t \%$ BS + $10 w t \%$ HS + $3 w t \%$ MAPP & 0.997 & 161.8 \\
\hline
\end{tabular}

\subsection{Mechanical Properties}

PP has different properties at various temperatures, which limits its technical applications. Specialist literature data show that the glass transition temperature of homopolymer PP is $-10{ }^{\circ} \mathrm{C}$ [21]. Below this temperature, the polymer becomes brittle and rigid. The influence of temperature on the mechanical properties change is presented in Figures 3-6. Tensile strength, tensile modulus, strain at break, and un-notched Charpy impact strength were determined. Results obtained from the test carried out at ambient temperature showed that the tensile strength increased by $56 \%$, and the tensile modulus increased by $74 \%$, for composites with $10 \mathrm{wt} \%$ fillers. The highest tensile strength was observed for composites with $20 \mathrm{wt} \%$ fillers (54.2 MPa). An increase in the temperature caused PP to become more ductile; consequently, tensile strength and tensile modulus decreased at $80^{\circ} \mathrm{C}$. The addition of fillers caused a significant decrease in elongation; however, reinforced composites did not show significant differences in strain at break at various temperatures. The highest values were obtained for composites tested at $-24{ }^{\circ} \mathrm{C}$. Composites reinforced by lignocellulosic fillers were characterized by low strength properties and an increase of tensile modulus. Demirer et al. [22] studied the usability of ground HSs in PP matrix composites. The addition of ground HSs caused a decrease in tensile strength and an increase in Young's modulus. Tensile strength for composites with $20 \mathrm{wt} \%$ HSs was $27.2 \mathrm{MPa}$, and tensile modulus was $1925 \mathrm{MPa}$. Composites reinforced with BFs had better mechanical properties. Tensile strength and tensile modulus was 70.7 MPa and 6114 MPa, respectively, for composites with $23 \mathrm{wt} \% \mathrm{BFs}$ [23]. In our study, the addition of the two kinds of fillers caused an increase in both tensile strength and tensile modulus. However, mechanical properties increased primarily due to the addition of BFs.

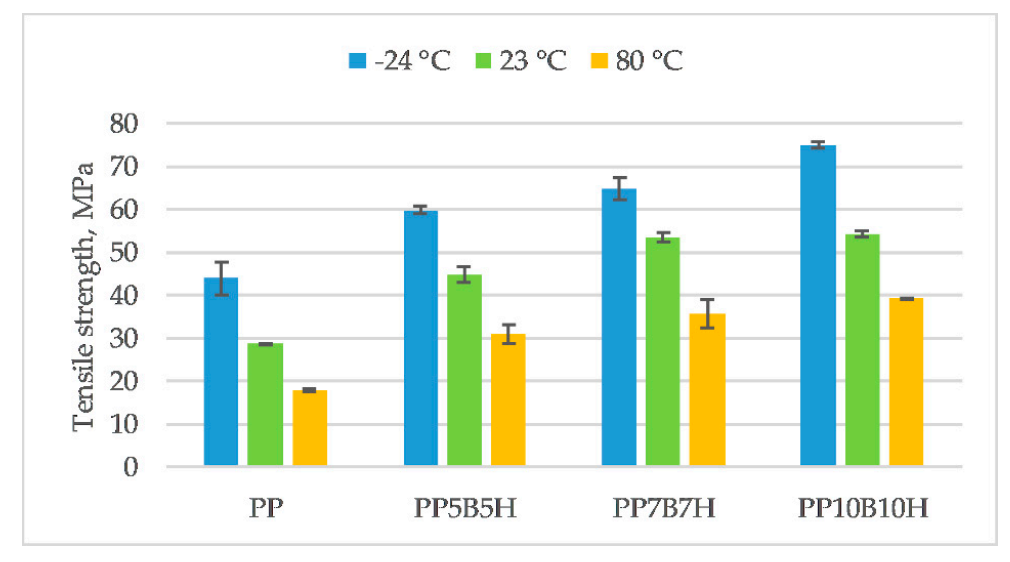

Figure 3. Tensile strength of the tested materials at $-24,23$, and $80{ }^{\circ} \mathrm{C}$. 


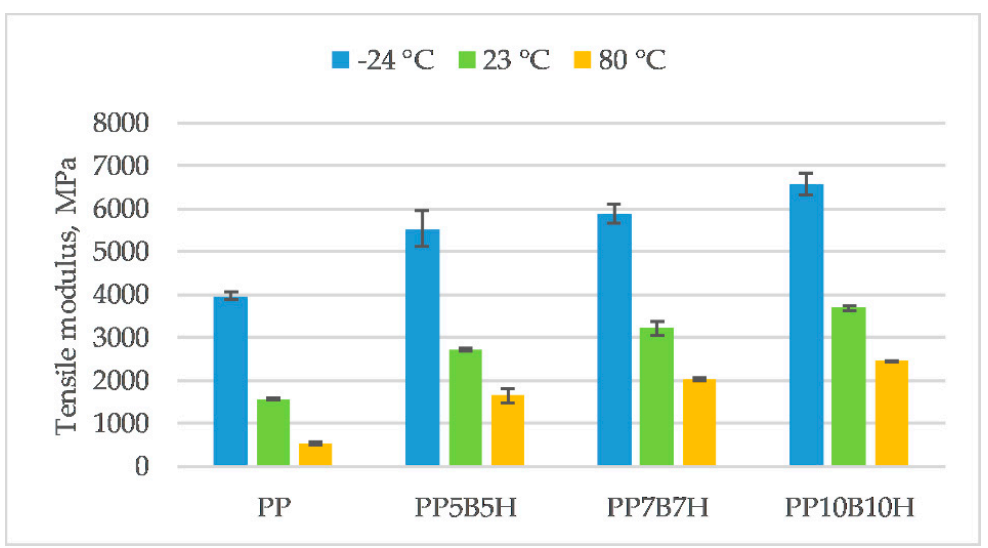

Figure 4. Tensile modulus of the tested materials at $-24,23$, and $80{ }^{\circ} \mathrm{C}$.

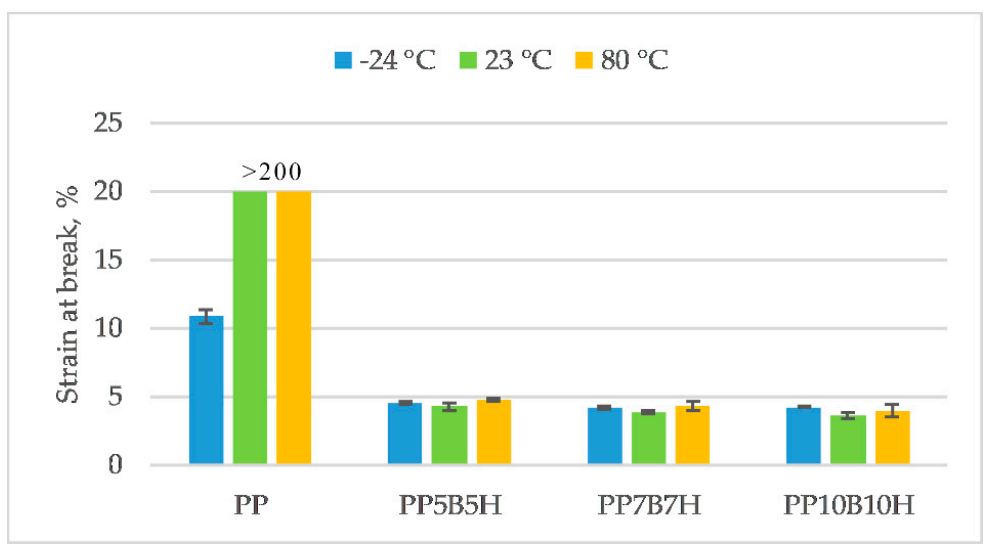

Figure 5. Strain at break of the tested materials at $-24,23$, and $80^{\circ} \mathrm{C}$.

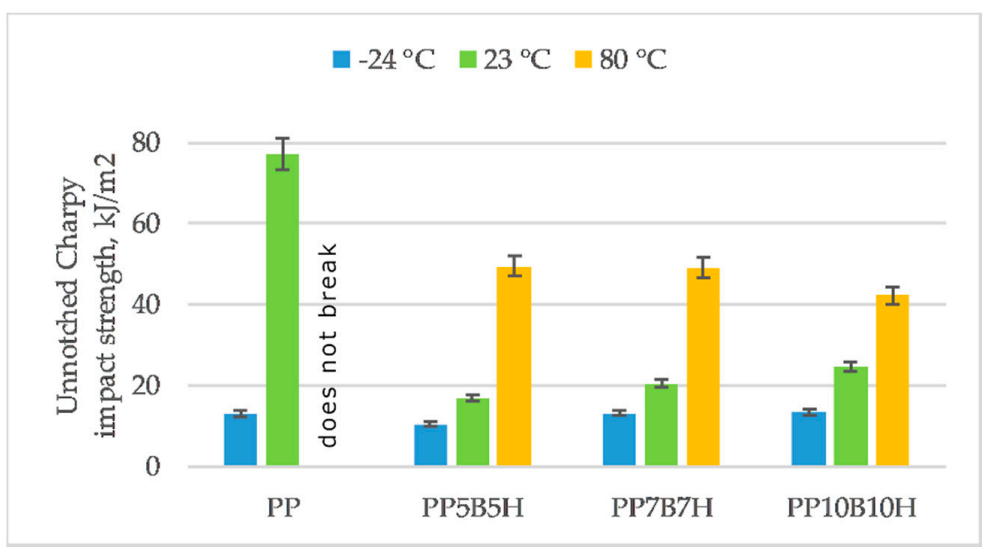

Figure 6. Un-notched Charpy impact strength of tested materials at $-24,23$, and $80^{\circ} \mathrm{C}$.

The Charpy impact test measures the amount of energy absorbed by a composite during fracture. Neat PP is characterized by high impact strength. The addition of fillers caused a significant decrease of the impact strength; however, further addition of fillers up to $20 \mathrm{wt} \%$ affected an increase of impact strength. This increase of the impact strength is caused by the pull-out of fibers and the friction on the interphase. This was expected because HS is a relatively hard material and it could result in a decrease of the impact strength. The impact strength also depends on the pull-out of fibers, matrix fracture, and debonding of fibers and matrix. At lower temperatures, composites become more brittle and the impact toughness is low. At $-24^{\circ} \mathrm{C}$, unfilled PP exhibited an $83 \%$ drop in impact strength. An addition of $10 \mathrm{wt} \%$ fillers caused a lower drop in impact strength at $-24{ }^{\circ} \mathrm{C}$, which was $37 \%$. An increase in 
temperature caused that material to become more ductile; as a consequence, the impact toughness was higher. Unfiled PP at elevated temperatures did not break and composites with 10, 15, and $20 \mathrm{wt} \%$ fillers increased by $193 \%, 140 \%$, and $42 \%$, respectively.

\subsection{Thermal Conductivity and Coefficient of Thermal Expansion}

Figure 7 shows the thermal conductivity of the tested composites. Thermal conductivity is the capacity of materials to transfer heat from one point to another. PP is characterized by high resistance to electricity and can be classed as a good isolator. The addition of fibers caused an increase of thermal conductivity. The highest value was observed for composites with $20 \mathrm{wt} \%$ fillers, where thermal conductivity increased by $8 \%$. Idicula et al. [24] studied the effect of fiber treatment on thermal conductivity. They examined whether sodium hydroxide treatment of fibers increased thermal conductivity. The addition of treated HSs could increase the thermal conductivity of hybrid composites.

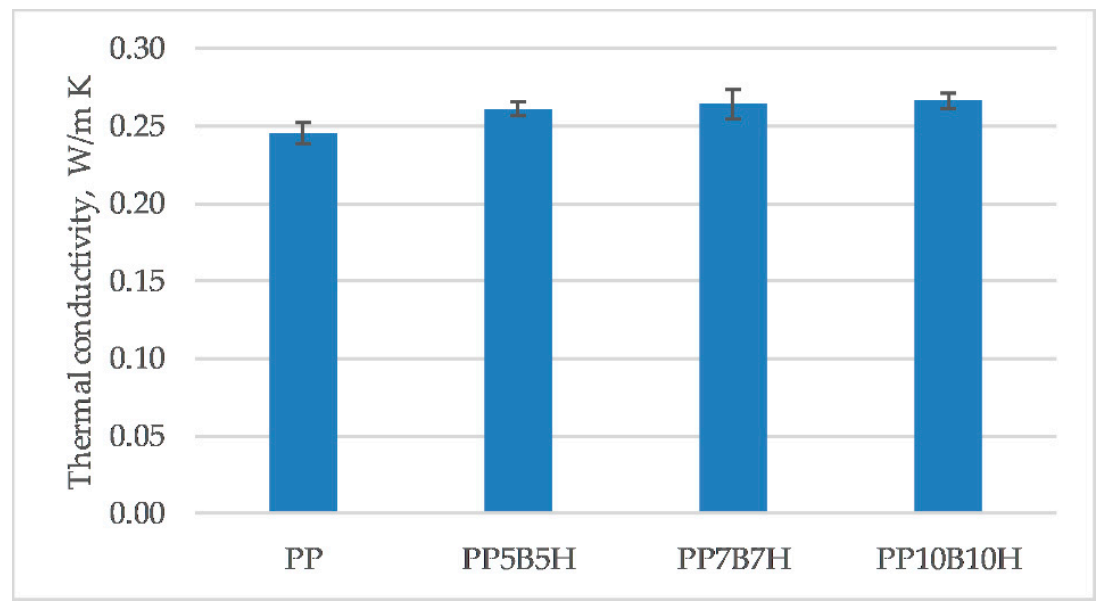

Figure 7. Thermal conductivity of the tested composites.

Figure 8 presents thermal curves of the tested composite. Table 2 presents LCTE at temperatures from -20 to $50{ }^{\circ} \mathrm{C}$. To achieve dimensional stability, a low LCTE is required. The linear coefficient decreased for all composites. It decreased by approximately 150\% and 200\% for PP5B5H and PP10B10H, respectively. The use of HS and BF helped to lower the overall LCTE values for hybrid composites.

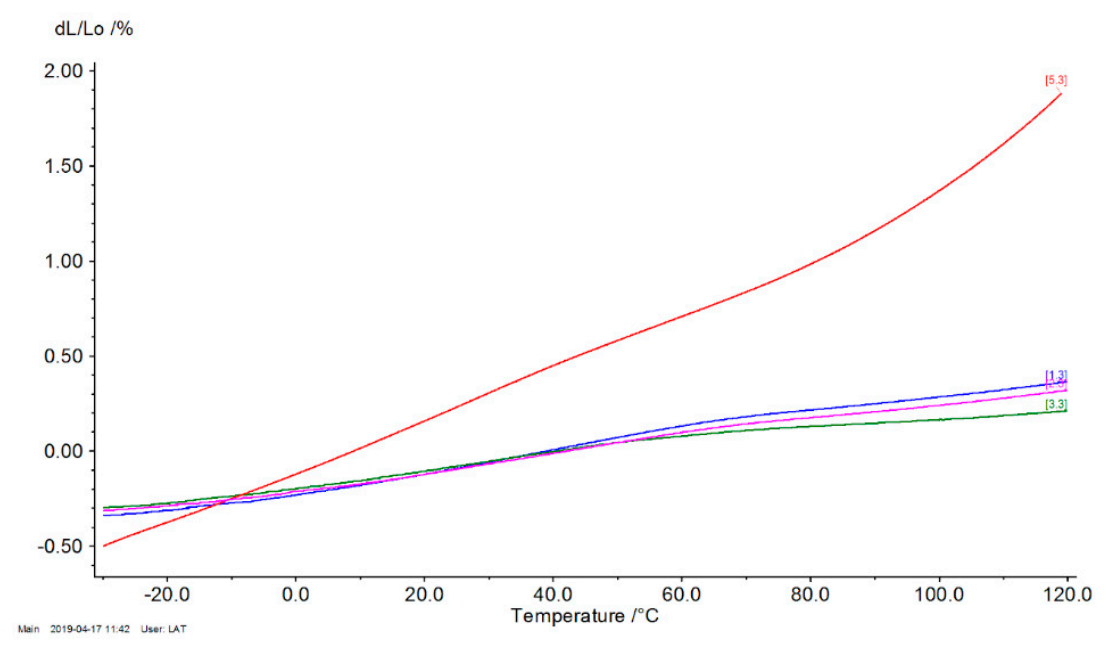

Figure 8. Thermal expansion curves of the tested composites: red line-polypropylene (PP), blue line-PPB5H, pink line-PP7B7H, and green line-PP10B10H. 
Table 2. Linear coefficient of thermal expansion of tested materials.

\begin{tabular}{cc}
\hline Specimen & Linear Coefficient of Thermal Expansion $\left(-\mathbf{2 0}\right.$ to $\left.\mathbf{5 0}{ }^{\circ} \mathbf{C}\right) \boldsymbol{\alpha} \times \mathbf{1 0}^{-\mathbf{6}} \mathbf{, ~} \mathbf{~ / K}$ \\
\hline PP & 136.6 \\
PP5B5H & 54.8 \\
PP7B7H & 47.4 \\
PP10B10H & 45.8 \\
\hline
\end{tabular}

\subsection{Creep Test}

When composites are exposed to a long-lasting dead load, the creep behavior becomes a critical parameter. The creep affects the dimensional stability of composites. Figure 9 presents the effect of an addition of fillers on the creep behavior of composites. PP exhibits other creep-like thermoplastic materials. As the fillers content increased, the creep strain was reduced. This behavior showed a noticeable improvement in the creep stability of the hybrid composites that was induced by the presence of rigid BFs and HSs.

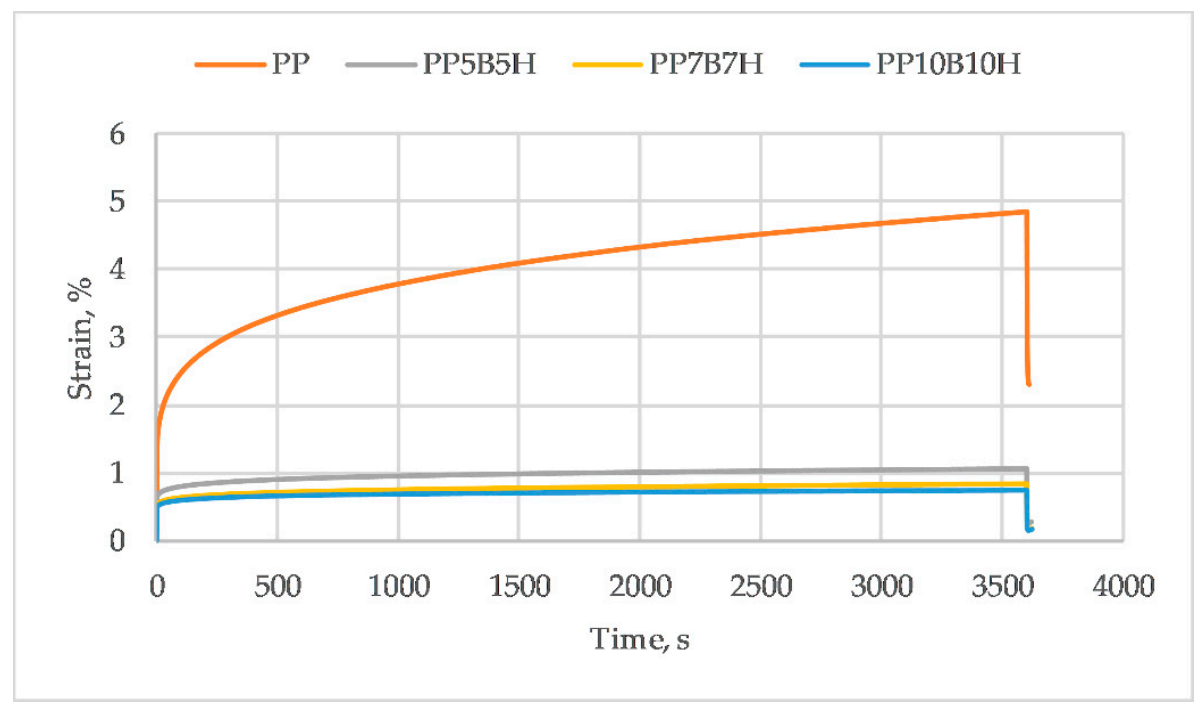

Figure 9. The effects of basalt fibers (BF) and hazelnut shell (HS) application level on the creep behavior of composites.

\subsection{Fractographic Investigation}

The morphology of the fractured surfaces of tensile specimens was observed by the used SEM and optical microscope. In Figure 10, we can observe HS particles of different sizes. BFs are not clearly visible at this magnification. Figure 10a presents a composite with $5 \mathrm{wt} \%$ BFs and $5 \mathrm{wt} \%$ HSs. HS particles are not uniformly distributed, and, in this magnified fragment, less of them can be detected, in contrast to the composite with $10 \mathrm{wt} \% \mathrm{BFs}$ and $10 \mathrm{wt} \% \mathrm{HSs}$.

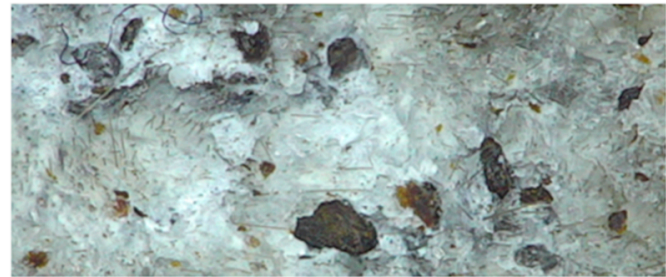

a)

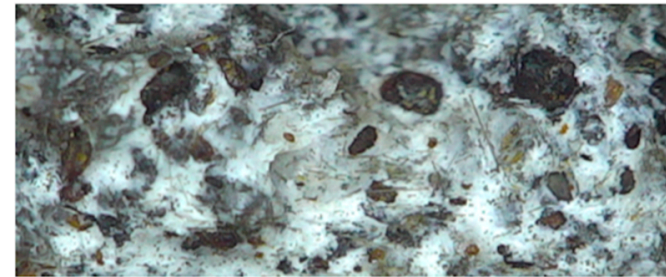

b)

Figure 10. Optical micrographs of tensile fractured surfaces of (a) PP5B5H and (b) PP10B10H with 20× magnification. 
From the observations of SEM images (Figure 11), it may be concluded that HS particles formed agglomerates in a polymer matrix, which contributes to the deterioration of mechanical properties. The dispersion of HS in PP can be improved by the addition of a promoter of mixing. Furthermore, HSs can be used in the form of granules. BFs were observed with the measured diameter of $17 \mu \mathrm{m}$. Hazelnut shell is a lignocellulosic material with an irregular surface. The adhesion between HS particles, BFs, and the matrix was strong; however, there were voids around the BFs and fiber pullouts are noticeable. HS particles are well-embedded in the polymer matrix. In Figure 11a, ductile fracture occurs. In Figure 11b, a larger amount of pulled fibers is noticeable. This resulted in increased impact strength and decreased mechanical properties.
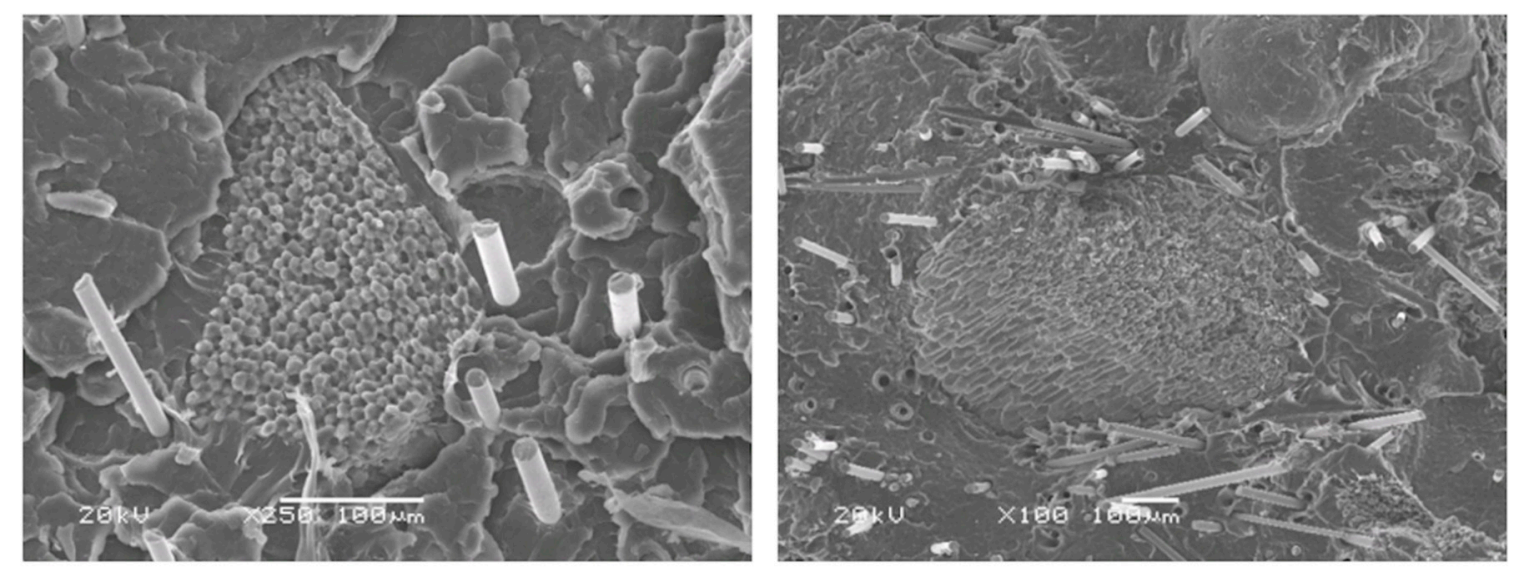

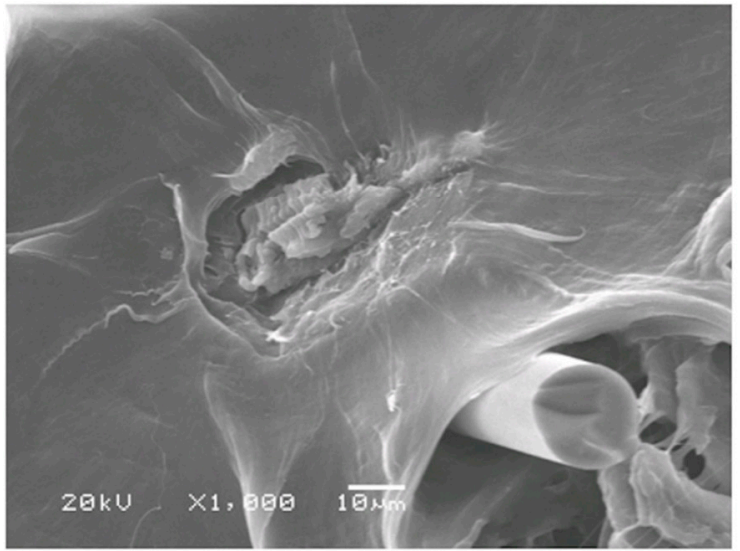

a)

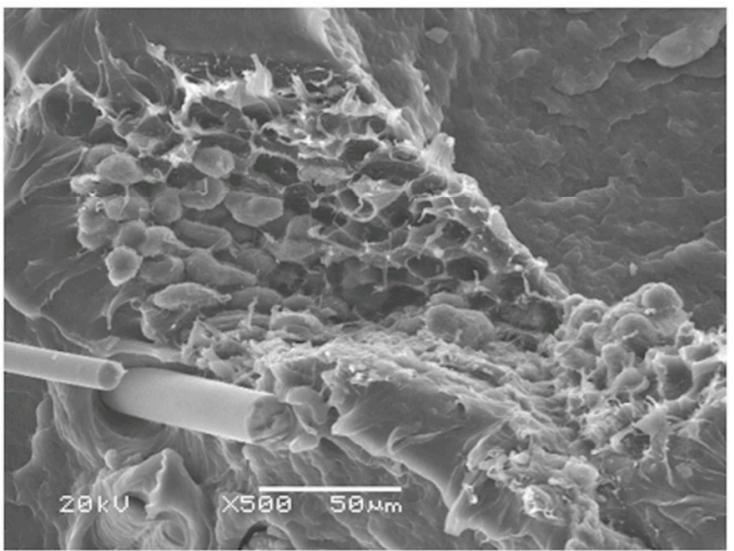

b)

Figure 11. Scanning electron microscope (SEM) micrographs of tensile fractured surfaces of (a) PP5B5H and (b) PP10B10H.

\subsection{The Influence of Water Absorption and Thermal Aging}

Figure 12 presents the water absorption of the tested materials. Neat PP had a very low water absorption of about $0.03 \%$. Water absorption increased with a growing filler content; however, the introduction of BFs in hybrid composites provided improved water absorption behavior. The increase of water absorption may be caused by an increased interfacial void between BF and the matrix, and because of the hydrophilic HS porous structure, which could accommodate water. This leads to a bad fiber/matrix interface, which results in a reduction in the mechanical properties of the composites. MAPP as a coupling agent is commonly used to improve fiber/matrix interface properties, and hence it reduces water absorption [25,26].

Figures 13-15 compare the changes in tensile strength, tensile modulus, and strain at break, before and after they were subjected to the 30-day incubation in water, and after $144 \mathrm{~h}$ of aging. 
After incubation in water, the tensile strength decreased, which was expected due to the addition of lignocellulosic filler. Moreover, the water absorption would weaken the interfacial bonding between the fillers and polymer. Water absorption causes debonding of the interfacial adhesion between the filler and the matrix. After incubation in water, PP10B10H composites showed a reduction in tensile strength by approximately $7 \%$. The effect of water absorption on tensile properties of hybrid composites was not significant. The tensile modulus increased for composites; it could be a result of swelling HS particles after the water absorption. Swelling could increase the contact area between the HS and the matrix.

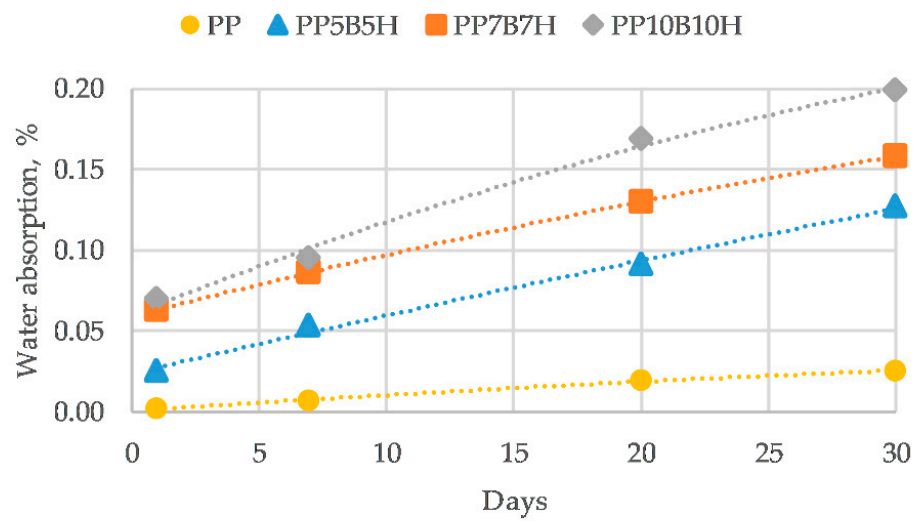

Figure 12. Water absorption of the tested materials.

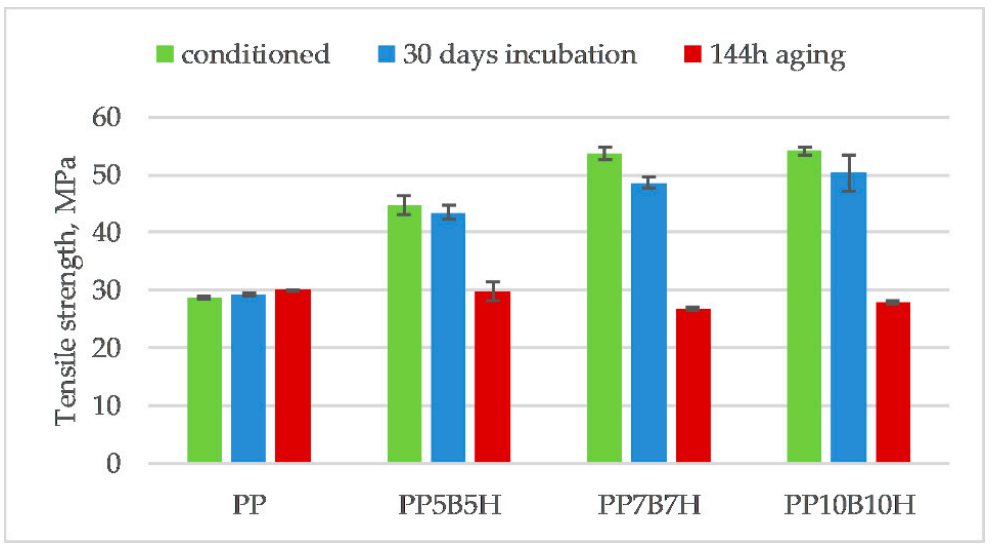

Figure 13. Comparison of tensile strength of PP-based composites before and after 30-day incubation in water and $144 \mathrm{~h}$ of aging.

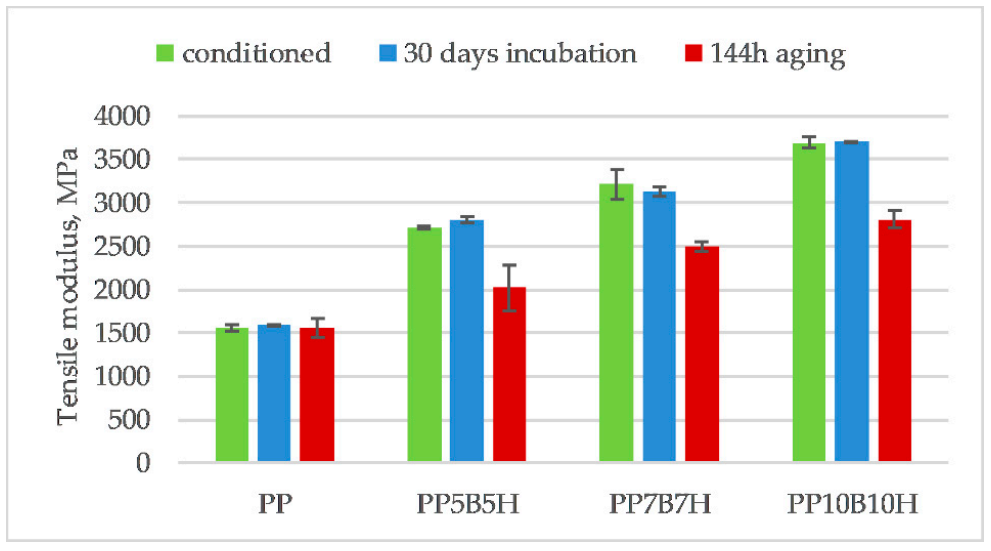

Figure 14. Comparison of tensile modulus of PP-based composites before and after 30-day incubation in water and $144 \mathrm{~h}$ of aging. 


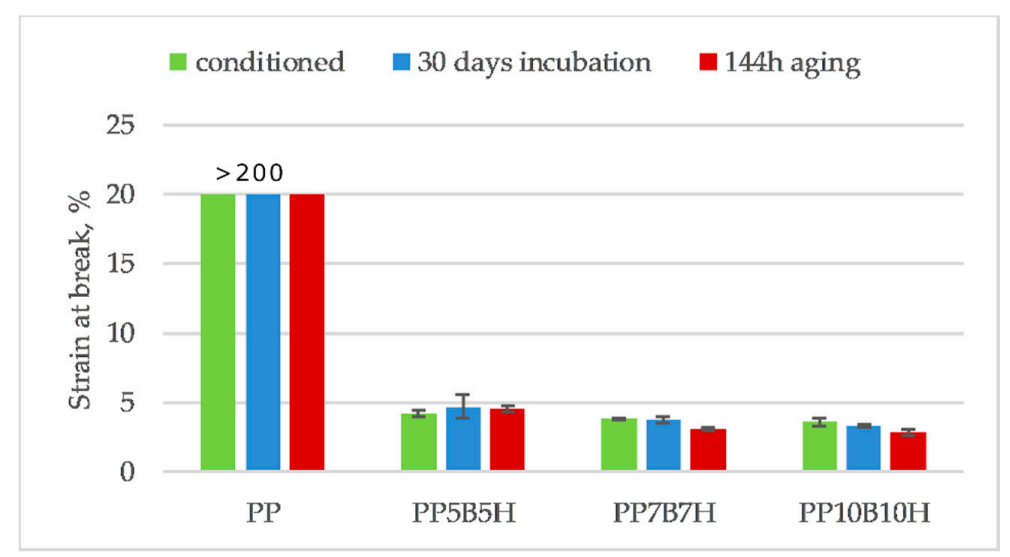

Figure 15. Comparison of strain at break of PP-based composites before and after 30-day incubation in water and $144 \mathrm{~h}$ of aging.

A composite's behavior during service conditions, analyzed by conducting aging, is one of the critical factors that affects its end use. The processes occurring in the materials during aging had an adverse effect on their strength properties. After aging, the tensile strength and tensile modulus decreased. There was no significant effect of aging on the tensile strength for unfilled PP. For composites with 10 and $20 \mathrm{wt} \%$ fillers, various physical and chemical factors can cause irreversible structural changes to occur in polymers, which cause a decrease in molecular weight (degradation) or a change in the chemical composition (destruction). Table 3 shows the weight changes before and after aging. The tested composites reduced weight, except for neat PP.

Table 3. The weight changes of the tested composites before and after aging.

\begin{tabular}{ccccc}
\hline & PP & PP5B5H & PP7B7H & PP10B10H \\
\hline \multicolumn{3}{c}{ g } \\
\hline Before drying & 36.78 & 32.9 & 34.13 & 34.91 \\
After drying at $70^{\circ} \mathrm{C}$ & 36.79 & 32.84 & 33.96 & 34.71 \\
Percentage weight difference & $-0.03 \%$ & $0.18 \%$ & $0.50 \%$ & $0.57 \%$ \\
\hline
\end{tabular}

\section{Conclusions}

The main conclusion is that HSs can be used as a filler in hybrid PP composites. HS-reinforced composites are characterized by low strength properties. However, the addition of both BFs and HSs caused an increase in tensile strength and tensile modulus. The tensile strength increased by $56 \%$ and the modulus of elasticity increased by $74 \%$ for composites already reinforced by $10 \mathrm{wt} \%$ of fillers. The results showed that addition of fillers successfully improved the thermal and creep stability. The effect of water absorption on mechanical properties of composites was not significant. After thermal aging, the tensile strength and modulus of elasticity significantly decreased. SEM micrographs showed the morphology of HSs and BFs. Debonding and pull-out of fibers, noticed for composites with $20 \mathrm{wt} \%$ of fillers, caused a decrease in strength properties and an increase in impact strength. To improve the adhesion between the BFs and the matrix, further studies are planned.

Author Contributions: Conceptualization, S.K.; methodology, S.K.; validation, S.K.; formal analysis, A.K.; investigation, A.K.; resources, S.K.; writing-original draft preparation, A.K.; writing-review and editing, A.K. and S.K.; visualization, A.K.; supervision, S.K. All authors have read and agreed to the published version of the manuscript.

Funding: This research received no external funding.

Acknowledgments: The authors thank Grupa Azoty, Poland, for their contribution to the preparation of test specimens. 
Conflicts of Interest: The authors declare no conflicts of interest.

\section{References}

1. Bledzki, A.K.; Mamun, A.A.; Erdmann, K.; Volk, J. Characterization of Grain By-Products and Properties of Its Biodegrade Composites. In Proceedings of the Naro.Tech Messe und Kongresse fü Nachwa Chsende Rohstoffe, Erfurt, Germany, 6-9 September 2007; pp. 1-11. Available online: http://www.escm.eu.org/docs/ eccm13/2615.pdf (accessed on 15 November 2019).

2. Fuad, M.Y.A.; Mustafah, J.; Mansor, M.S.; Ishak, Z.A.M.; Omar, A.K.M. Thermal properties of polypropylene/rice husk ash composites. Polym. Int. 1995, 38, 33-43. [CrossRef]

3. Chun, K.S.; Husseinsyah, S.; Osman, H. Utilization of cocoa pod husk as filler in polypropylene biocomposites: Effect of maleated polypropylene. J. Thermoplast. Compos. Mater. 2015, 28, 1507-1521. [CrossRef]

4. García-García, D.; Carbonell, A.; Samper, M.D.; García-Sanoguera, D.; Balart, R. Green composites based on polypropylene matrix and hydrophobized spend coffee ground (SCG) powder. Compos. Part B Eng. 2015, 78, 256-265. [CrossRef]

5. Factfish. Available online: http://www.factfish.com/ (accessed on 12 November 2019).

6. Çöpür, Y.; Güler, C.; Akgül, M.; Taşçioğlu, C. Some chemical properties of hazelnut husk and its suitability for particleboard production. Build. Environ. 2007, 42, 2568-2572. [CrossRef]

7. Gurbanov, N.A.; Abbasov, I.I.; Ismayilova, K.H.; Hasanova, N.A. Production of Polypropylene Matrix Polymer Composites with Hazelnut Shell Fillings. Phys. Mech. Prop. 2018, 16, 559-565.

8. Balart, J.F.; Garc, D.; Balart, R.; Boronat, T. Manufacturing and Properties of Biobased Thermoplastic Composites From Poly (lactid acid) and Hazelnut Shell Wastes. Polym. Compos. 2018, 39, 848-857. [CrossRef]

9. Vikas, G.; Sudheer, M. A Review on Properties of Basalt Fiber Reinforced Polymer Composites. Am. J. Mater. Sci. 2017, 7, 156-165.

10. Bunsell, A.R. Handbook of Properties of Textile and Technical Fibres; Woodhead Publishing: Cambridge, UK, 2018; ISBN 9781782424581.

11. Sang, L.; Zheng, G.; Hou, W.; Yang, X.; Wei, Z. Crystallization and mechanical properties of basalt fiber-reinforced polypropylene composites with different elastomers. J. Therm. Anal. Calorim. 2018, 134, 1531-1543. [CrossRef]

12. Greco, A.; Maffezzoli, A.; Casciaro, G.; Caretto, F. Mechanical properties of basalt fibers and their adhesion to polypropylene matrices. Compos. Part B Eng. 2014, 67, 233-238. [CrossRef]

13. Botev, M.; Betchev, H.; Bikiaris, D.; Panayiotou, C. Mechanical Properties and Viscoelastic Behavior of Basalt. J. Appl. Polym. Sci. 1999, 74, 523-531. [CrossRef]

14. Manikandan, V.; Winowlin Jappes, J.T.; Suresh Kumar, S.M.; Amuthakkannan, P. Investigation of the effect of surface modifications on the mechanical properties of basalt fibre reinforced polymer composites. Compos. Part B Eng. 2012, 43, 812-818. [CrossRef]

15. Yang, W.; Jia, Z.; Chen, Y.; Zhang, Y.; Si, J.; Lu, H.; Yang, B. Carbon nanotube reinforced polylactide/basalt fiber composites containing aluminium hypophosphite: Thermal degradation, flame retardancy and mechanical properties. RSC Adv. 2015, 5, 105869-105879. [CrossRef]

16. Swolfs, Y.; Gorbatikh, L.; Verpoest, I. Fibre hybridisation in polymer composites: A review. Compos. Part A Appl. Sci. Manuf. 2014, 67, 181-200. [CrossRef]

17. Mazur, K.; Kuciel, S.; Salasinska, K. Mechanical, fire, and smoke behaviour of hybrid composites based on polyamide 6 with basalt/carbon fibres. J. Compos. Mater. 2019, 53, 3979-3991. [CrossRef]

18. Samal, S.K.; Mohanty, S.; Nayak, S.K. Banana/glass fiber-reinforced polypropylene hybrid composites: Fabrication and performance evaluation. Polym. Plast. Technol. Eng. 2009, 48, 397-414. [CrossRef]

19. Guo, G.; Finkenstadt, V.L.; Nimmagadda, Y. Mechanical properties and water absorption behavior of injection-molded wood fiber/carbon fiber high-density polyethylene hybrid composites. Adv. Compos. Hybrid Mater. 2019, 1-11. Available online: https://link.springer.com/article/10.1007/s42114-019-00116-5 (accessed on 15 November 2019). [CrossRef]

20. Franciszczak, P.; Kalniňš, K.; Błędzki, A.K. Hybridisation of man-made cellulose and glass reinforcement in short-fibre composites for injection moulding-Effects on mechanical performance. Compos. Part B Eng. 2018, 145, 14-27. [CrossRef] 
21. Karger-Kocsis, J.; Bárány, T. Polypropylene Handbook; Springer International Publishing: Cham, Switzerland, 2019; Volume 43, ISBN 978-3-030-12902-6.

22. Demirer, H.; Kartal, İ; Yıldırım, A.; Büyükkaya, K. The Utilisability of Ground Hazelnut Shell as Filler in Polypropylene Composites. Acta Phys. Pol. A 2018, 134, 254-257. [CrossRef]

23. Kufel, A.; Kuciel, S. Study on mechanical properties of basalt fibre reinforced polypropylene composites. In Modern Polymeric Materials For Environment Applications; Pielichowski, K., Ed.; Tomasz Majka Publisher: Tarnów, Poland, 2019; pp. 229-237.

24. Idicula, M.; Boudenne, A.; Umadevi, L.; Ibos, L.; Candau, Y.; Thomas, S. Thermophysical properties of natural fibre reinforced polyester composites. Compos. Sci. Technol. 2006, 66, 2719-2725. [CrossRef]

25. Dobreva, D.; Nenkova, S.; Vasileva, S. Morphology and mechanical properties of polypropylene-wood flour composites. BioResources 2006, 1, 209-219.

26. Franco-Marquès, E.; Méndez, J.A.; Pèlach, M.A.; Vilaseca, F.; Bayer, J.; Mutjé, P. Influence of coupling agents in the preparation of polypropylene composites reinforced with recycled fibers. Chem. Eng. J. 2011, 166, 1170-1178. [CrossRef]

(C) 2019 by the authors. Licensee MDPI, Basel, Switzerland. This article is an open access article distributed under the terms and conditions of the Creative Commons Attribution (CC BY) license (http://creativecommons.org/licenses/by/4.0/). 